\title{
Sums of squares in function fields of hyperelliptic curves
}

\author{
Karim Johannes Becher • Jan Van Geel
}

\begin{abstract}
We study sums of squares, quadratic forms, and related field invariants in a quadratic extension of the rational function field in one variable over a hereditarily pythagorean base field.
\end{abstract}

\section{Introduction}

Let be given a field $k$ such that the arithmetic properties of $k$ and of its finite extensions are well understood. It is natural to consider arbitrary finitely generated cxtensions $F / k$ of transcendence degree $n$ and to study the arithmetic properties of $F$. A satisfactory approach to this general problem is given by Tsen-Lang Theory in the cases where $k$ is algebraically closed or finite. The arithmetic properties of fields addressed in this article are those related to sums of squares and quadratic forms. Important field invariants in this context are the pythagoras number and the $u$-invariant. In the case where $k$ is a real closed field there are important results on the values of these invariants for function fields $F / k$, due to Pfister and to Elman and Lam (see [12, Chaps. 7, 8] for a survey). These results, however, have also highlighted some outstanding open problems.

The pythagoras number is the least upper bound on the number of squares necessary to express an arbitrary element of a field that is positive at all field orderings as a sum of squares. Assume that there is a common finite upper bound for the pythagoras number of all finite extensions of the field $k$. Then for any function field in one variable $F / k$ the pythagoras number of $F$ has to be finite (see (3.6) below). A special case where this applies is the situation where the ground field $k$ is hereditarily pythagorean. However, even in this case it

\section{K. J. Becher (ख)}

Universität Konstanz, FB Mathematik und Statistik, D203, 78457 Konstanz, Germany

e-mail: becher@maths.ucd.ie

J. Van Geel

Universiteit Gent, Vakgroep Zuivere Wiskunde en Computeralgebra, Galglaan 2,

9000 Gent, Belgium

e-mail: jvg@cage.ugent.be 
is an open problem whether there is a common upper bound on the pythagoras numbers of all function fields in one variable $F / k$.

In characteristic different from 2, Milnor's Exact Sequence (cf. [9, Chap. IX, Sect. 3]) relates the quadratic form theory of the rational function field $k(X)$ to the quadratic form theory of all finite extensions of $k$. For instance, this leads to Becker's result (see (4.1) below) that $k$ is hereditarily pythagorean if and only if the pythagoras number of $k(X)$ equals 2 .

The results contained in this article are mostly concerned with function fields of hyperelliptic curves, that is, with quadratic extensions of a rational function field. The techniques we use are based on the relations between quadratic forms over the two fields of a quadratic field extension.

In [16] it is shown that, if $k=\mathbb{R}((t))$ and if $F$ is the function field of a hyperelliptic curve $C$ with good reduction, then $p(F)=2$. The proof is based on the calculation of the Brauer group of the curve $C$. This work inspired us to look at the problem from a purely quadratic form theoretic point of view. Apart from giving a different proof for the main result in [16], we obtain several results for function fields of hyperelliptic curves over a hereditarily pythagorean field in general. As a consequence of these results, given a function field $F$ of a hyperelliptic curve over a field $k$ "like $\mathbb{R}((t))$ "-namely carrying a henselian valuation $k \rightarrow \mathbb{Z} \cup\{\infty\}$ with hereditarily euclidean residue field - we are able to decide now in many cases whether $p(F)$ is either 2 or larger than 2. Moreover, in several of those cases we can also prove that the $u$-invariant of such a field $F$ is 4 , by extending a method sketched in [16, p. 307], which before was only applicable in the cases where $p(F)=2$.

All fields considered in this article are assumed to be of characteristic different from 2 . In fact, most results on function fields $F / k$ obtained here are interesting only in the case where at least the ground field $k$ is real, and in this case all fields involved are of characteristic zero. All quadratic forms occurring in this article are tacitly understood to be regular.

We shall assume that the reader is familiar with the basic theory of quadratic forms over fields. References for this theory are [9,14]. All information needed on function fields in one variable is largely covered by [15], for example.

\section{Preliminaries}

Let $K$ be a field of characteristic different from 2. The set of nonzero sums of squares in $K$ is denoted by $\sum K^{2}$. Given a field $K$, we denote by $W K$ the Witt ring of $K$ and by $I K$ its fundamental ideal, and we put $I^{n} K=(I K)^{n}$ for $n \in \mathbb{N}$. The torsion ideal in $W K$ is denoted by $W_{t} K$. Recall that a quadratic form $\varphi$ over $K$ is torsion if it represents a torsion element in the Witt ring $W K$, which by Pfister's Local-Global Principle (cf. [9, Chap. VIII, Sect. 3]) is the case if and only if $\varphi$ has trivial signature with respect to every ordering of $K$. Given a (regular) quadratic form $\varphi$ over $K$, we denote by $D_{K}(\varphi)$ the set of nonzero elements of $K$ represented by $\varphi$. We say that a quadratic form $\varphi$ over $K$ is universal if $D_{K}(\varphi)=K^{\times}$.

2.1 Field invariants in quadratic form theory

For $x \in K$, we denote by $\ell_{K}(x)$ the least positive integer $r$ such that $x$ can be written as a sum of $r$ nonzero squares in $K$ if such $r$ exists, otherwise we put $\ell_{K}(x)=\infty$. With $r \in \mathbb{N}$ we write $D_{K}(r)=\left\{x \in K^{\times} \mid \ell_{K}(x) \leq r\right\}$. Hence $\sum K^{2}=\bigcup_{r \in \mathbb{N}} D_{K}(r)=\left\{x \in K^{\times} \mid \ell_{K}(x)<\infty\right\}$. The number

$$
p(K)=\sup \left\{\ell_{K}(x) \mid x \in \sum K^{2}\right\} \in \mathbb{N} \cup\{\infty\}
$$

is called the pythagoras number of $K$. If $p(K)=1$, then $K$ is said to be pythagorean. The value 


$$
s(K)=\ell_{K}(-1)
$$

is called the level of $K$. Note that $\ell_{K}(0)=s(K)+1 \geq p(K)$, with the usual conventions for calculating with $\infty$. By the Artin-Schreier Criterion [9, Chap. VIII, (1.11)], $s(K)=\infty$ holds if and only if $K$ admits a field ordering; if this is the case, the field $K$ is said to be real, otherwise nonreal. A famous result due to Pfister says that the level of a nonreal field is always a power of 2 and that any 2-power is the level of some field (cf. [9, Chap. XI, (2.2) and (2.6)]). If $K$ is a nonreal field, then $s(K) \leq p(K) \leq s(K)+1$, by [9, Chap. XI, (5.6)], so in particular $p(K) \in\left\{2^{n}, 2^{n}+1 \mid n \in \mathbb{N}\right\}$. For the real case, Hoffmann showed in [7] that any positive integer is the pythagoras number of some uniquely ordered field.

The $u$-invariant of $K$ is defined as

$$
u(K)=\sup \{\operatorname{dim}(\varphi) \mid \varphi \text { anisotropic torsion form over } K\} \in \mathbb{N} \cup\{\infty\} .
$$

This definition of the $u$-invariant was introduced by Elman and Lam in [4]. Whenever $u(K)$ is finite, it equals the least integer $n$ such that every torsion form over $K$ of dimension strictly larger than $n$ is isotropic. Furthermore, by [9, Chap. XIII, (6.28)] $p(K) \leq u(K)$ holds for any field $K$ except if $K$ is real pythagorean, in which case $p(K)=1$ and $u(K)=0$.

Both, the pythagoras number and the $u$-invariant of a given field are in general difficult to determine. For many interesting fields it is not even known whether these invariants are finite. For an account on known results about these field invariants we refer to the corresponding chapters of [12].

The following is a variation of [1, (3.5)]. It will turn out to be useful especially for showing that certain fields have $u$-invariant equal to 4 .

Theorem 2.1 Assume that $I^{3} K$ is torsion free. Let $d \in \sum K^{2}$ be an element such that $\left|\sum K^{2} / D_{K}(\langle 1, d\rangle)\right| \leq 2$. Then $u(K) \leq u(K(\sqrt{-d}))$.

Proof Let $L=K(\sqrt{-d})$. For arbitrary $a, b \in \sum K^{2}$, we claim that the quadratic form $\varphi=\langle 1, d,-a,-b\rangle$ over $K$ is universal. If $\varphi$ is isotropic, this is trivial. Suppose that $\varphi$ is anisotropic. Then

$$
\emptyset=D_{K}(\langle 1, d\rangle) \cap D_{K}(\langle a, b\rangle)=D_{K}(\langle 1, d\rangle) \cap a D_{K}(\langle 1, a b\rangle) .
$$

Since $D_{K}(\langle 1, a b\rangle) \subseteq \sum K^{2}$ and $\left|\sum K^{2} / D_{K}(\langle 1, d\rangle)\right| \leq 2$, it follows that $a \notin D_{K}(\langle 1, d\rangle)$ and $D_{K}(\langle 1, a b\rangle) \subseteq D_{K}(\langle 1, d\rangle)$. This yields the inclusion $D_{K}(\langle 1, a b,-a,-b\rangle) \subseteq D_{K}(\varphi)$. But $\langle 1, a b,-a,-b\rangle$ is a torsion 2-fold Pfister form over $K$, thus universal by the assumption that $I^{3} K$ is torsion free. Then $\varphi$ is universal, too.

Let now $\alpha$ be an arbitrary anisotropic torsion form over $K$ of $\operatorname{dimension} \operatorname{dim}(\alpha)>4$. We claim that $\alpha$ remains anisotropic over $L$. Suppose on the contrary that $\alpha_{L}$ is isotropic. Then by [9, Chap. VII, (3.1)], $\alpha$ contains up to scaling the norm form $\langle 1, d\rangle$ of the quadratic extension $L / K$. Without loss of generality, we may thus assume that $\alpha=\langle 1, d\rangle \perp \alpha^{\prime}$. If $K$ is nonreal, then the above claim yields that $\alpha$ is isotropic over $K$, in contradiction to the choice of $\alpha$. Assume now that $K$ is real. Since $I^{3} K$ is torsion free, using [2, (3.5) and (4.2)] we can write $\alpha^{\prime}=\beta \perp \gamma$ over $K$ where $\gamma$ is a torsion form and $\beta$ is a form such that $m \times \beta$ is anisotropic for any $m \in \mathbb{N}$. Since $\alpha=\langle 1, d\rangle \perp \beta \perp \gamma$ and $\gamma$ are torsion forms over $K$, the same holds for $\langle 1, d\rangle \perp \beta$. Since $d \in \sum K^{2}$ and by the property of $\beta$, it follows that $\operatorname{dim}(\beta)=2$ and $\beta=\langle-a,-b\rangle$ for certain $a, b \in \sum K^{2}$. Then $\alpha=\langle 1, d,-a,-b\rangle \perp \gamma$, so the above claim yields a contradiction to the assumptions that $\alpha$ anisotropic and $\operatorname{dim}(\alpha)>4$.

This argument shows that $u(L) \geq u(K)$ whenever $u(K)>4$. It remains to consider the cases where $u(L)<4$. Recall that $u(L) \neq 3$. Assume now that $u(L) \leq 2$. Then $I^{2} L=0$, 
which by [9, Chap. XI, (4.1) and (4.7)] implies that $I^{2} K$ is torsion free and thus $u(K) \leq 2$. Finally, if $u(L)=1$, then $K$ is pythagorean, whence $u(K) \leq 1$.

We shall use the notation $K(i)=K(\sqrt{-1})$.

Corollary 2.2 If $\left|\sum K^{2} / D_{K}(2)\right| \leq 2$ and $u(K(i))<8$, then $u(K) \leq u(K(i))$.

Proof If $u(K(i))<8$, then $I^{3} K(i)=0$ and thus $I^{3} K$ is torsion free, by [9, Chap. XI, (4.4) and (4.7)]. Therefore, the statement follows from (2.1).

2.2 Values of Pfister forms over quadratic extensions

Given elements $a_{1}, \ldots, a_{n} \in K^{\times}$we denote by $\left\langle\left\langle a_{1}, \ldots, a_{n}\right\rangle\right\rangle$ the $n$-fold Pfister form $\left\langle 1,-a_{1}\right\rangle \otimes \cdots \otimes\left\langle 1,-a_{n}\right\rangle$ over $K$. Given an $n$-fold Pfister form $\pi$ over $K$, we write $\pi^{\prime}$ for its pure part and $\pi^{(a)}=\langle a\rangle \perp \pi^{\prime}$. We refer to the 0 -fold Pfister form $\langle 1\rangle$ as the trivial Pfister form.

Theorem 2.3 Let $a \in K$ and $L=K(\sqrt{a})$ and let $\pi$ be a nontrivial Pfister form defined over $K$. Then

$$
K^{\times} \cap D_{L}(\pi)=D_{K}(\pi) \cdot D_{K}\left(\pi^{(a)}\right) .
$$

Proof Obviously, $K^{\times} \cap D_{L}(\pi) \supseteq D_{K}(\pi) \cdot D_{K}\left(\pi^{(a)}\right)$. To show the converse inclusion, let $b \in K^{\times} \cap D_{L}(\pi)$. Then the Pfister form $\rho=\pi \otimes\langle 1,-b\rangle$ is hyperbolic over $L$. Therefore, the form $\rho$ considered over $K$ contains a binary subform $\langle c,-a c\rangle$ with $c \in K^{\times}$. (In fact, if $\rho$ is isotropic over $K$, then it is hyperbolic and thus contains $\langle 1,-a,-1, a\rangle$.) Then $c \in D_{K}(\rho)$, whence $c \rho \cong \rho$ and thus $\langle 1,-a\rangle \subseteq c \rho=\rho$. Witt Cancellation now shows that $\rho^{\prime}$ represents $-a$, i.e. $\rho^{(a)}$ is isotropic over $K$. Since $\rho^{(a)}=\pi^{(a)} \perp-b \pi$, the fact that this form is isotropic just means that $b \in D_{K}(\pi) \cdot D_{K}\left(\pi^{(a)}\right)$.

At least for 1 -fold Pfister forms (2.3) is common knowledge.

Corollary 2.4 Let $a, b \in K$ and $L=K(\sqrt{a})$. Then

$$
K^{\times} \cap D_{L}(\langle 1, b\rangle)=D_{K}(\langle 1, b\rangle) \cdot D_{K}(\langle 1, a b\rangle) .
$$

Proof By (2.3), we have $K^{\times} \cap D_{L}(\langle 1, b\rangle)=D_{K}(\langle 1, b\rangle) \cdot D_{K}(\langle a, b\rangle)$. As $D_{K}(\langle a, b\rangle)=$ $b D_{K}(\langle 1, a b\rangle)$ and $b D_{K}(\langle 1, b\rangle)=D_{K}(\langle 1, b\rangle)$, the statement follows.

For later use, we explicitly state the following special case.

Corollary 2.5 Let $a \in K$ and $L=K(\sqrt{a})$. Then

$$
K^{\times} \cap D_{L}(2)=D_{K}(2) \cdot D_{K}(\langle 1, a\rangle) .
$$

Proof This is what (2.4) yields for $b=1$.

2.3 Ramification of torsion forms

Given a discrete rank-one valuation $w: K \longrightarrow \mathbb{Z} \cup\{\infty\}$ with residue field $\kappa$ of characteristic different from 2 and an element $u \in K$ of valuation $u(u)=1$, one defines the first and second residue homomorphisms $\partial_{1}, \partial_{2}: W K \longrightarrow W_{K}$ with respect to $w$ and $u$ (see [9, p. 147]). The quadratic forms over $\kappa$, determined up to Witt equivalence, that represent the images under 
$\partial_{1}$ and $\partial_{2}$ of a given form over $K$ are called its first and second residue forms, respectively. A quadratic form over $K$ is said to be unramified in $w$ if its Witt equivalence class lies in the kernel of $\partial_{2}$, otherwise it is said to be ramified in $w$. Actually, the kernel of $\partial_{2}$ and therefore the notion of being ramified in $w$ depend on the valuation $w$, but not on the choice of the uniformiser $u$.

We are essentially concerned with the $k$-valuations of the rational function field $E=k(X)$ over a real field $k$. Apart from the degree valuation, any such valuation is associated to a (unique) monic irreducible polynomial $f \in k[X]$, and this is our canonical choice for the uniformising element for this valuation. We say that a quadratic form over $k(X)$ is unramified over $k$, if it is unramified in all $k$-valuations of $k(X)$.

Lemma 2.6 Let $k$ be a real field and $\varphi$ a torsion form over $k(X)$. Let $w$ be a $k$-valuation on $k(X)$ with real residue field $\kappa_{w}$. If $n \in \mathbb{N}$ is such that $p\left(\kappa_{w}\right) \leq 2^{n}$, then $2^{n} \times \varphi$ is unramified at $w$.

Proof Since $p\left(\kappa_{u j}\right) \leq 2^{n}$, the torsion part of $W \kappa_{u}$ is annihilated by $2^{n}$ (cf. [9, Chap. XI, (5.6)]). Therefore, the second residue homomorphism $\partial_{2}: W k(X) \rightarrow W \kappa_{w}$ with respect to $w$ is trivial on $2^{n} \cdot W_{t} k(X)$. If $f$ is irreducible, then we may say that $f$ is real or nonreal, respectively, if the root field $k[X] / f(X)$ is real or nonreal. It follows from [9, Chap. $\mathrm{X},(2.13)]$ that a monic irreducible polynomial $f \in k[X]$ is nonreal if and only $f$ is a sum of squares in $k(X)$.

Lemma 2.7 Let $k$ be a real field. Assume that $n \in \mathbb{N}$ is such that $p(K) \leq 2^{n}$ holds for any finite real extension $K / k$. Let $\varphi$ be a torsion form over $k(X)$. Then the form $2^{n} \times \varphi$ over $k(X)$ is either hyperbolic, or it is ramified at some $k$-valuation associated to a nonreal monic irreducible polynomial in $k[X]$.

Proof Assume that $2^{n} \times \varphi$ is unramified at every $k$-valuation of $E=k(X)$ that is associated to a nonreal monic irreducible polynomial. Observe that the residue field of the degree valuation is the real field $k$. Using the previous lemma, it then follows that $2^{n} \times \varphi$ is unramified over $k$. So, by Milnor's Exact Sequence (cf. [9, p. 306]), there exists a form $\rho$ over $k$ such that $2^{n} \times \varphi=\rho_{E}$.

We claim that $\rho$ is Witt equivalent to $2^{n} \times \psi$ for some form $\psi$ over $k$. As for $n=0$ there is nothing to show, we may assume $n \geq 1$. Let $\pi=2^{n} \times\langle 1\rangle$. Since $\rho_{E}=2^{n} \times \varphi$, the form $\rho$ becomes hyperbolic over $E(\pi)$, the function field of $\pi$ over $E$. On the other hand, $E(\pi)$ is a rational function field in one variable over $k(\pi)$, and thus the scalar extension $W k(\pi) \longrightarrow W E(\pi)$ is injective. Therefore, $\rho$ becomes hyperbolic already over $k(\pi)$. As the kernel of the scalar extension map $W k \longrightarrow W k(\pi)$ is the ideal generated by $\pi$ (cf. [14, Chap. 4, (5.4)]), it follows that $\rho$ is Witt equivalent to $\pi \otimes \psi=2^{n} \times \psi$ for some form $\psi$ over $k$, as we claimed.

Now, since $\rho_{E}=2^{n} \times \varphi$ is a torsion form over $E$, it follows that $\rho$ and $\psi$ are torsion forms over $k$. However, the hypothesis contains that $p(k) \leq 2^{n}$, so that $W_{t} k$ is $2^{n}$-torsion. Therefore, $2^{n} \times \psi$ and $\rho$ are hyperbolic over $k$. Thus $2^{n} \times \varphi=\rho_{E}$ is hyperbolic over $E=k(X)$.

\section{Pythagoras numbers of function fields}

By a function field in one variable over $k$ we mean a finitely generated field extension $F / k$ of transcendence degree one where $k$ is relatively algebraically closed in $F$.

Lemma 3.1 If $F / k$ is a finitely generated, nonalgebraic extension, then $p(F) \geq 2$. 
Proof Let $n$ be the transcendence degree of $F / k$. Then $n \geq 1$ and $F$ is $k$-isomorphic to a finite extension of $E=k\left(X_{1}, \ldots, X_{n}\right)$. Now, $E$ is not pythagorean, because $1+X_{1}^{2}$ is not a square in $E$. The Diller-Dress Theorem [9, Chap. VIII, (5.7)] then asserts that $F$ is not pythagorean either.

Proposition 3.2 Let $f \in k[X, Y]$ be an irreducible polynomial that is not constant in $Y$ and let $n=\operatorname{deg}_{Y}(f)$. Let $F$ be the quotient field of $k[X, Y] /(f)$. Then $2 \leq p(F) \leq n \cdot p(k(X))$.

Proof The first inequality is valid by (3.1), and the second follows from the fact that $p(L) \leq$ $[L: K] \cdot p(K)$ holds for any finite field extension $L / K$ (see [12, p. 103]).

For certain function fields in one variable $F / k$ one can bound $p(F)$ from below by $p(k(X))$.

Proposition 3.3 Let $F / k$ be a function field in one variable. If $F$ has a divisor of odd degree, then $p(F) \geq p(k(X))$ and $u(F) \geq u(k(X))$.

Proof If there exists a divisor of odd degree over $F$, then there is a $k$-valuation $v$ on $F$ such that, with $F_{v}$ denoting the residue field, the residue degree $\left[F_{v}: k\right]$ is odd. Let $g$ denote the genus of $F$. We choose an odd number $m$ with $(m-1) \cdot\left[F_{v}: k\right] \geq 2 g$. We compare the divisors $D=m \cdot v$ and $D^{\prime}=(m-1) \cdot v$. For $f \in F$ let $(f)$ denote the principal divisor over $F$ defined by $f$. For any divisor $C$ on $F$ we put $\mathcal{L}(C)=\{f \in F \mid(f) \geq-(C)\} \cup\{0\}$. Since both divisors $D$ and $D^{\prime}$ are of degree larger than $2 g-2$, the Riemann-Roch theorem yields that $\operatorname{dim}(\mathcal{L}(D))=\operatorname{deg}(D)-g+1$ and $\operatorname{dim}\left(\mathcal{L}\left(D^{\prime}\right)\right)=\operatorname{deg}\left(D^{\prime}\right)-g+1$, showing that $\mathcal{L}\left(D^{\prime}\right) \subsetneq \mathcal{L}(D)$. We choose $z \in \mathcal{L}(D) \backslash \mathcal{L}\left(D^{\prime}\right)$. Then $(z) \geq-m \cdot v$ while $(z) \nsucceq-(m-1) \cdot v$, which implies that $v$ is the only pole of $z$, and that this pole is of order $-v(z)=m$. By $[15$, Chap. I, (4.11)], we have $[F: k(z)]=\left[F_{v}: k\right] \cdot m$, which is an odd number. Therefore, Springer's Theorem [9, Chap. VII, (2.7)] implies that $p(F) \geq p(k(z))$ as well as $u(F) \geq$ $u(k(z))$. Obviously, $k(z)$ is $k$-isomorphic to $k(X)$.

Remark 3.4 In quadratic form theory, there are several interesting invariants $i$ of fields $K$ that are of the type

$$
i(K)=\sup \{\operatorname{dim}(\varphi) \mid \varphi \text { anisotropic form over } K \text { satisfying } \mathcal{P}\} \in \mathbb{N} \cup\{\infty\},
$$

where $\mathcal{P}$ is a certain property of quadratic forms that is conserved under scalar extension (cf. [8, Sect. 3]). The $u$-invariant and the pythagoras number are examples of such invariants, corresponding to the property of being a torsion form and the property of having a diagonalisation $\langle 1, \ldots, 1,-s\rangle$ with $s$ a sum of squares, respectively. Since the argument in the proof of (3.3) is based on Springer's Theorem for quadratic forms and odd degree extensions, under the same hypothesis on $F / k$ the same proof yields the inequality $i(F) \geq i(k(X))$ for any field invariant $i$ of this type.

The last two statements relate the pythagoras number of a general function field in one variable $F / k$ to that of the rational function field $k(X)$. The next theorem relates the pythagoras number of $k(X)$ to sums of squares in finite extensions of $k$. It extends Pfister's result [11, Satz 2].

By a 2-extension we mean an arbitrary separable algebraic field extension $F / E$ such that the Galois group of the Galois closure of this extension is a 2-group. A finite 2-extension $F / E$ is characterised by the existence of a tower of fields $E=F_{0} \subseteq F_{1} \subseteq \cdots \subseteq F_{r}=F$ where $F_{i} / F_{i-1}$ is a quadratic extension for $i=1, \ldots, r$. 
Theorem 3.5 Let $k$ be a real field and $n \in \mathbb{N}$. The following are equivalent:

(i) $p(K)<2^{n+1}$ for any finite real extension $K / k$.

(ii) $s(K) \leq 2^{n}$ for any finite nonreal extension $K / k$.

(iii) $p(k(X)) \leq 2^{n+1}$.

Proof See [11, Satz 2] and [9, p. 397] for the equivalence (ii $\Leftrightarrow$ iii).

(ii $\Rightarrow i$ ) Assume $K / k$ is a finite real extension with $p(K) \geq 2^{n+1}$. So there is some element $a \in \sum K^{2}$ with $\ell_{K}(a)=2^{n+1}$. Then $K(\sqrt{-a})$ is a finite nonreal extension of $k$ with $s(K(\sqrt{-a}))=2^{n+1}$.

$(i \Rightarrow i i)$ Assume that $K / k$ is a finite nonreal extension with $s(K)>2^{n}$. Since the level of a nonreal field is a power of 2 , this means that $s(K) \geq 2^{n+1}$. Let $M$ be the normal closure of $K / k$. Let $S$ be a 2-Sylow subgroup of $\operatorname{Gal}(M / k)$ that contains a 2-Sylow subgroup $S^{\prime}$ of $\operatorname{Gal}(M / K)$. Let $L=M^{S}$, the fixed field of $S$ in $M$. Then $L / k$ is of odd degree and therefore $L$ is a real field, too. Since $K L$ is fixed by $S^{\prime}$, the extension $K L / K$ has odd degree, and therefore $s(K L)=s(K) \geq 2^{n+1}$. Moreover, $M / L$ is a 2-extension. As $K L \subseteq M$, it follows that $K L / L$ is a finite 2-extension, so there is a finite tower of fields $L=K_{0} \subseteq K_{1} \subseteq \cdots \subseteq K_{r}=K L$ where $K_{i} / K_{i-1}$ is a quadratic extension for $i=1, \ldots, r$. Since $L$ is real while $K L$ is nonreal, we can choose $m \in\{1, \ldots, r\}$ such that $K_{m-1}$ is real and $K_{m}$ nonreal. We write $K_{m}=K_{m-1}(\sqrt{-a})$ with $a \in \sum K_{m-1}{ }^{2}$. Since $s\left(K_{m}\right) \geq s(K L) \geq 2^{n+1}$, we conclude that $\ell_{K_{m-1}}(a) \geq 2^{n+1}$. Therefore, $K_{m-1}$ is a finite real extension of $k$ with $p\left(K_{m-1}\right) \geq 2^{n+1}$.

Remark 3.6 If $k$ is a field such that there is a common finite upper bound on the pythagoras numbers of all finite extensions of $k$, then $p(F)$ is finite for every function field in one variable $F / k$. In fact, if $k$ is nonreal this is trivial, and in the real case this follows from (3.5) and (3.2). This fact is probably known, but we could not find a reference for it.

Given $f \in k[X]$ over a field $k$, we say that a root $\alpha$ of $f$ is real or nonreal, if the field $k(\alpha)$ has the corresponding property.

Proposition 3.7 Let $k$ be a field and $n \in \mathbb{N}$ such that $s(K) \leq 2^{n}$ holds for any finite nonreal extension of $K / k$. Let $F$ be the function field of $Y^{2}=f(X)$ over $k$ where $f \in k[X]$ is a monic polynomial with only nonreal roots. Then $p(F) \leq 2^{n+1}$.

Proof We may assume that $k$ is real. By (3.5) we have $p(k(X)) \leq 2^{n+1}$. It follows from the assumptions on the polynomial $f$ that it is a sum of squares in $k(X)$. Therefore, $F=$ $k(X)(\sqrt{f})$ is a totally positive quadratic extension of $k(X)$. Then $p(k(X)) \leq 2^{n+1}$ implies that $p(F) \leq 2^{n+1}$ (cf. [5, (2.18)]).

Using (3.5) we obtain the following technical lemma, which will turn out to be useful in the study of quadratic extensions of a rational function field.

Lemma 3.8 Let $k$ be a real field and let $n \in \mathbb{N}$ be such that $p(K)<2^{n+1}$ for any finite real extension $K / k$. Let $F=k(X)(\sqrt{f})$ with $f \in k[X]$. Then

$$
\sum F^{2}=D_{F}\left(2^{n+1}\right) \cdot\left(k(X)^{\times} \cap \sum F^{2}\right) .
$$

Moreover, any class in $\sum F^{2} / D_{F}\left(2^{n+1}\right)$ is represented by a polynomial $g \in k[X]$ with only real roots.

Proof Let $E=k(X)$. By the hypothesis and (3.5), we have $p(E) \leq 2^{n+1}$. Let $\xi \in \sum F^{2}$. Then $N_{F / E}(\xi) \in \sum E^{2}=D_{E}\left(2^{n+1}\right)$. By the Norm Principle $([5,(2.13)])$, we have $\xi \in$ 
$E^{\times} \cdot D_{F}\left(2^{n+1}\right)$, so there is some $h \in E^{\times}$such that $\xi \cdot D_{F}\left(2^{n+1}\right)=h \cdot D_{F}\left(2^{n+1}\right)$. Obviously, we can choose such $h$ in $k[X]$, and we additionally obtain that $h \in \sum F^{2}$. We now factorise $h=g \cdot h^{\prime}$ where $g, h^{\prime} \in k[X]$ are such that $g$ has only real roots while $h^{\prime}$ is monic and has only nonreal roots. Then $h^{\prime} \in \sum E^{2}=D_{E}\left(2^{n+1}\right)$ and therefore $\xi \cdot D_{F}\left(2^{n+1}\right)=h \cdot D_{F}\left(2^{n+1}\right)=$ $g \cdot D_{F}\left(2^{n+1}\right)$.

Proposition 3.9 Let $k$ be a real field. Assume that $n \in \mathbb{N}$ is such that $p(K) \leq 2^{n}$ for any finite real extension $K / k$. Let $F=k(X)(\sqrt{f})$ where $f \in k[X]$ is a nonzero squarefree polynomial. Let $g \in k[X]$ be a nonzero polynomial with only real roots. Then $g \in$ $D_{k(X)}\left(2^{n} \times\langle 1, f\rangle\right)$ if and only if $g \in \sum F^{2}$ and $g(\alpha) \in D_{k(\alpha)}\left(2^{n}\right)$ for every nonreal root $\alpha$ of $f$.

Proof Note that $D_{k(X)}\left(2^{n} \times\langle 1, f\rangle\right) \subseteq \sum F^{2}$. Hence, for both directions we may assume that $g \in \sum F^{2}$. Consider the form $\varphi=\langle 1, f,-g,-f g\rangle$ over $E=k(X)$. This is a torsion form, because $g \in E^{\times} \cap \sum F^{2}=\sum E^{2}+f \sum E^{2}$. Now, $g \in D_{E}\left(2^{n} \times\langle 1, f\rangle\right)$ if and only if $2^{n} \times \varphi$ is hyperbolic over $E$, and by (2.7) this is equivalent to having that $2^{n} \times \varphi$ is unramified at every $k$-valuation associated to a nonreal monic irreducible polynomial in $k[X]$. Moreover, since $g$ has only real roots, the only nonreal monic irreducible polynomials in which $2^{n} \times \varphi$ might possibly be ramified are the factors of $f$. So, $g \in D_{E}\left(2^{n} \times\langle 1, f\rangle\right)$ if and only if $2^{n} \times \varphi$ is unramified at $p$ for every minimial polynomial $p$ of a nonreal root $\alpha$ of $f$. Now given such $\alpha$ and $p$, by definition the second residue homomorphism $\partial_{2}$ with respect to the valuation given by $p$ maps the class of $2^{n} \times \varphi$ in $W E$ to the class of a form similar to $2^{n} \times\langle 1,-g(\alpha)\rangle$ in $W k(\alpha)$; this class is trivial if and only if $g(\alpha) \in D_{k(\alpha)}\left(2^{n}\right)$.

Theorem 3.10 Let $k$ be a real field and $n \in \mathbb{N}$ such that $p(K) \leq 2^{n}$ holds for any finite real extension $K / k$. Let $F=k(X)(\sqrt{f})$ where $f \in k[X]$ is a nonzero square-free polynomial. Let $K_{1}, \ldots, K_{r}$ denote the root fields of the distinct nonreal irreducible factors of $f$. Then $p(F) \leq 2^{n+2}$ and

$$
\left|\sum F^{2} / D_{F}\left(2^{n+1}\right)\right| \leq \prod_{i=1}^{r}\left|K_{i}^{\times} / D_{K_{i}}\left(2^{n}\right)\right| .
$$

In particular, if $f$ has only real roots, then $p(F) \leq 2^{n+1}$.

Proof By (3.5) the hypothesis implies that $p(k(X)) \leq 2^{n+1}$, and then $p(F) \leq 2^{n+2}$ follows by (3.2).

Let $f_{1}, \ldots, f_{r}$ be the distinct nonreal monic irreducible factors of $f$ and $\alpha_{1}, \ldots, \alpha_{r}$ corresponding roots, so that $K_{i}=k\left(\alpha_{i}\right)$ for $1 \leq i \leq r$. By (3.8), any class of $\sum F^{2} / D_{F}\left(2^{n+1}\right)$ is represented by a polynomial $g \in k[X]$ with only real roots. By (3.9), for such $g$ we have $g \in D_{k(X)}\left(2^{n} \times\langle 1, f\rangle\right) \subseteq D_{F}\left(2^{n+1}\right)$ provided that $g\left(\alpha_{i}\right) \in D_{K_{i}}\left(2^{n}\right)$ for $1 \leq i \leq r$. We thus can define an injective homomorphism $\sum F^{2} / D_{F}\left(2^{n+1}\right) \rightarrow \prod_{i=1}^{r} K_{i}^{\times} / D_{K_{i}}\left(2^{n}\right)$ by sending the class of a polynomial $g$ as above to $\left(g\left(\alpha_{1}\right) D_{K_{1}}\left(2^{n}\right), \ldots, g\left(\alpha_{r}\right) D_{K_{r}}\left(2^{n}\right)\right)$. This yields the bound on $\left|\sum F^{2} / D_{F}\left(2^{n+1}\right)\right|$. Moreover, if $f$ has only real roots, then we obtain that $\sum F^{2}=D_{F}\left(2^{n+1}\right)$, i.e. $p(F) \leq 2^{n+1}$.

\section{Hereditarily pythagorean fields}

A field $k$ is said to be hereditarily pythagorean if $k$ is real and if any finite real extension of $k$ is pythagorean. Such fields have been characterised by Becker [3] in various ways. In 
[3, Chap. III, Theorem 1] Becker showed that a real field $k$ is hereditarily pythagorean if and only if the absolute Galois group of $k(i)$ is abelian. The following characterisations of hereditarily pythagorean fields are contained in [3, Chap. III, Theorems 1, 4], and they can be retrieved from (3.5).

Theorem 4.1 (Becker) For a real field $k$ the following are equivalent:

(i) $k$ is hereditarily pythagorean.

(ii) Every finite nonreal extension of $k$ contains $k(i)$.

(iii) $p(k(X))=2$.

Proof This follows immediately from (3.5) and (3.1).

Corollary 4.2 Let $F$ be the function field of a hyperelliptic curve over a hereditarily pythagorean field. Then $2 \leq p(F) \leq 4$.

Proof By assumption, $F$ is $k$-isomorphic to a quadratic extension of $k(X)$, so the statement follows from (3.2) and (4.1).

Corollary 4.3 There exists a function field in one variable $F / k$ with a divisor of odd degree and with $p(F)=2$ if and only if either $k$ is hereditarily pythagorean or if $-1 \in k^{\times 2}$.

Proof Suppose $F$ is a function field in one variable over $k$ with $p(F)=2$ and having a divisor of odd degree. By (3.3) we have $2 \leq p(k(X)) \leq p(F)=2$, whence $p(k(X))=2$. In the case where $k$ is real, the statement thus follows from (4.1). On the other hand, if $k$ is nonreal, then $p(k(X))=2$ is equivalent with $-1 \in k^{\times 2}$. In fact, given any $a \in k^{\times}$, the element $a+X^{2}$ is not a sum of 2 squares in $k(X)$ unless $-1 \in k^{\times 2}$ or $a \in k^{\times 2}$, by the Second Representation Theorem [9, Chap. IX, (2.1)].

Question 4.4 Assume that $-1 \notin k^{\times 2}$ and that there exists a function field in one variable $F / k$ such that $p(F)=2$. Does this imply that $k$ is hereditarily pythagorean?

A field $K$ is called euclidean if it is pythagorean and uniquely ordered, which is actually equivalent to saying that $K^{\times 2} \cup\{0\}$ is a field ordering of $K$. If $K$ is real and every finite real extension of $K$ is euclidean, then $K$ is said to be hereditarily euclidean.

Theorem 4.5 (Elman-Wadsworth) Let $F / k$ be any function field in one variable. Then $u(F)=2$ if and only if $k$ is hereditarily euclidean or hereditarily quadratically closed. Otherwise $u(F) \geq 4$.

Proof Using (3.1), we have $2 \leq p(F) \leq u(F)$. Note that, on the one hand, $u(F) \leq 2$ is equivalent with $I^{2} F$ being torsion free, on the other hand, $k$ is (hereditarily) euclidean or (hereditarily) quadratically closed if and only if $k(i)$ is (hereditarily) quadratically closed. Hence, the claim follows from [6, Theorem] and the fact that $u(F) \neq 3[9$, Chap. XI, (6.8)].

Corollary 4.6 Let $k$ be hereditarily euclidean. Then $p(F)=u(F)=2$ for any function field in one variable $F / k$.

Proof This follows from the proof of (4.5).

We now obtain a characterisation of hereditarily euclidean fields. Note that the statement could also be obtained using Theorems 1 and 2 in [17]. 
Theorem 4.7 Let $F$ be the function field of the conic $X^{2}+Y^{2}+1=0$ over $k$. Then $p(F)=2$ if and only if either $-1 \in k^{\times 2}$ or $k$ is hereditarily euclidean. In any other case $p(F)=3$.

Proof By (3.1) and since $s(F) \leq 2$, we have $2 \leq p(F) \leq s(F)+1 \leq 3$. If $k$ is hereditarily euclidean, then (4.6) implies that $p(F)=2$. If $s(k) \leq 2$, then $F$ is actually a rational function field in one variable over $k$, and therefore $p(F)=s(k)+1$, and this corresponds to the claim.

Suppose now that $-1 \notin k^{\times 2}$ and $k$ is not pythagorean. Then we may choose an element $c \in k$ such that $1+c^{2} \notin k^{\times 2}$. Let $\zeta=c+\sqrt{-\left(1+X^{2}\right)} \in F$. From the Second Representation Theorem [9, Chap. IX, (2.1)], using that $\langle 1,1\rangle$ is anisotropic over $k$, we obtain that $N_{F / k(X)}(\zeta)=1+c^{2}+X^{2}$ is not a sum of 2 squares in $k(X)$. On the other hand, the norm of a sum of 2 squares is also a sum of 2 squares, by [9, Chap. VII, (5.1)]. Hence, $\zeta$ is not a sum of 2 squares in $F$.

So far, we have established the statement in particular for all cases where $k$ is either nonreal or hereditarily euclidean. Assume from now on that $k$ is real and not hereditarily euclidean. By [3, Chap. III, Lemma 5], then either $k$ is itself not euclidean, or it is not hereditarily pythagorean.

Suppose $k$ is not hereditarily pythagorean. Then there exists an algebraic element $\alpha$ over $k$ such that $k(\alpha)$ is real and $1+\alpha^{2}$ is not a square in $k(\alpha)$. Let $g \in k[X]$ be the minimal polynomial of $\alpha$ over $k$. We claim that $g$ is not a sum of 2 squares in $F$. Suppose on the contrary, that $g \in D_{F}(2)$. By (2.5), then $g \in D_{k(X)}(2) \cdot D_{k(X)}\left(\left\langle 1,-\left(1+X^{2}\right)\right\rangle\right)$. This means that there exists a polynomial $h \in k[X]$ with only nonreal roots such that the 2-fold Pfister form $\left\langle\left\langle\left(1+X^{2}\right), g h\right\rangle\right\rangle$ over $k(X)$ is hyperbolic. In particular, $\left\langle\left\langle\left(1+X^{2}\right), g h\right\rangle\right.$ is unramified with respect to the valuation associated to $g$. Since $g$ has a real root $\alpha$, it is clear that $h$ is not divisible by $g$. However, the second residue form of $\left\langle\left\langle\left(1+X^{2}\right), g h\right\rangle\right.$ with respect to $g$ is the form $\left\langle 1,-\left(1+\alpha^{2}\right)\right\rangle$ over $k(\alpha)$, and this form is anisotropic because $1+\alpha^{2} \notin k(\alpha)^{\times 2}$. This is a contradiction.

Suppose finally that $k$ is hereditarily pythagorean but not euclidean. Then there exists $t \in k^{\times}$such that $\pm t \notin k^{\times 2}=\sum k^{2}$. So the 2-fold Pfister form $\langle\langle-1, t\rangle\rangle$ is anisotropic and not isometric to $\langle\langle-1,-1\rangle$. Now, since $\langle\langle-1,-1\rangle\rangle$ is the only anisotropic 2-fold Pfister form over $k$ splitting over $F$, it follows that $\left\langle\langle-1, t\rangle\right.$ is anisotropic over $F$, whence $t \notin D_{F}(2)$.

The remainder of this section is focused on conditions on function fields of hyperelliptic curves $F / k$ that allow to conclude that $p(F)=2$.

Proposition 4.8 Let $k$ be a hereditarily pythagorean field. Let $f \in k[X]$ be a nonzero squarefree polynomial and $F=k(X)(\sqrt{f})$. Let $g \in k[X]$ be a nonzero polynomial with only real roots. Then $g \in D_{k(X)}(\langle 1, f\rangle)$ if and only if $g \in \sum F^{2}$ and $g(\alpha) \in k(\alpha)^{\times 2}$ for any nonreal root $\alpha$ of $f$.

Proof This is what (3.9) yields for $n=0$.

Corollary 4.9 Let $k$ be a hereditarily pythagorean field. Let $f \in k[X]$ be a nonzero squarefree polynomial and $F=k(X)(\sqrt{f})$. If $g(\alpha) \in k(\alpha)^{\times 2}$ holds for any nonreal root $\alpha$ of $f$ and any polynomial $g \in k[X] \cap \sum F^{2}$ with only real roots, then $p(F)=2$.

Proof Given any polynomial $g \in k[X] \cap \sum F^{2}$ with only real roots, using (4.8) we obtain that $g \in D_{k(X)}(\langle 1, f\rangle) \subseteq D_{F}(2)$. Therefore (3.8) applied with $n=0$ yields that $\sum F^{2}=D_{F}(2)$.

The following is a generalisation of [17, Theorem 3], the case where $F$ is real and the function field of a conic over $k$. 
Corollary 4.10 Let $k$ be a hereditarily pythagorean field. Let $F$ be the function field of the curve $Y^{2}=f(X)$ over $k$ where $f \in k[X]$. If $f$ has only real roots, or if $f$ is monic and has only nonreal roots, then $p(F)=2$.

Proof The first case follows from (4.9), the second case from (3.7).

A field $K$ is said to be $\infty$-pythagorean if $K^{2^{n}}+K^{2^{n}}=K^{2^{n}}$ holds for any $n \in \mathbb{N}$. We say that $K$ is hereditarily $\infty$ pythagorean if $K$ is real and if any finite real extension of $K$ is $\infty$-pythagorean. By [3, Chap. III, Theorem 9], a field $K$ is hereditarily $\infty$-pythagorean as soon as $L^{4}+L^{4}=L^{4}$ holds for any finite real extension $L / K$. A hereditarily pythagorean field does not need to be $\infty$-pythagorean, as a simple example in [3, p. 109] shows.

Lemma 4.11 Let $k$ be a hereditarily $\infty$-pythagorean field. Let $g \in k[X]$ be a polynomial with only real roots and such that $g \in \sum k(X)^{2}+X \sum k(X)^{2}$. Let $a, b \in k$. Then $g\left(a^{2} \pm b^{2} i\right) \in$ $k(i)^{2}$.

Proof Since $g$ is represented over $k(X)$ by a multiple of the form $\langle 1, X\rangle$, it follows from [12, Chap. 1, (2.3)] that $g=h_{1}+X h_{2}$ for certain polynomials $h_{1}, h_{2} \in k[X]$ that are sums of squares in $k[X]$. We may assume that $g$ is square-free, so that $h_{1}$ and $h_{2}$ are relatively prime. If we show that $g\left(a^{2}+b^{2} i\right)$ is a square in $k(i)$, then the same follows for its conjugate $g\left(a^{2}-b^{2} i\right)$. The leading coefficient of $g$ is a sum of squares in $k$, hence a square. It is therefore sufficient to show that $\widetilde{g}\left(a^{2}+b^{2} i\right) \in k(i)^{2}$ holds for any monic irreducible factor $\widetilde{g}$ of $g$.

Given such $\widetilde{g}$, we fix a root $\vartheta$ of $\widetilde{g}$ and observe that the field $K=k(\vartheta)$ is real and thus $\infty$-pythagorean. Note that $\tilde{g}(c)=N_{K(i) / k(i)}(c-\vartheta)$ for any $c \in k(i)$. Since $0=g(\vartheta)=$ $h_{1}(\vartheta)+\vartheta h_{2}(\vartheta)$ and both $h_{1}(\vartheta)$ and $h_{2}(\vartheta)$ are squares in $K$, we have $-\vartheta \in K^{2}$. Since $K$ is $\infty$-pythagorean, there exist $\gamma_{0}, \gamma_{1} \in K$ such that $2 \gamma_{0}^{2}=a^{2}-\vartheta$ and $\gamma_{1}^{4}=\gamma_{0}^{4}+\frac{1}{4} b^{4}$, and further $\beta_{0} \in K$ with $\beta_{0}^{2}=\gamma_{0}^{2}+\gamma_{1}^{2}$. If $\beta_{0}=0$, then $K$ being real implies that $a=b=$ $\vartheta=0$, whence $\widetilde{g}=X$ and $\widetilde{g}\left(a^{2}+b^{2} i\right)=0 \in k(i)^{2}$. Suppose now that $\beta_{0} \neq 0$ and put $\beta_{1}=\left(2 \beta_{0}\right)^{-1} b^{2} \in K$. Then $\beta_{0}^{4}-\frac{1}{4} b^{4}=2 \gamma_{0}^{2} \beta_{0}^{2}$ and thus

$$
\left(\beta_{0}+i \beta_{1}\right)^{2}=\left(\beta_{0}^{2}-\beta_{1}^{2}\right)+2 \beta_{0} \beta_{1} i=2 \gamma_{0}^{2}+b^{2} i=a^{2}+b^{2} i-\vartheta
$$

whence $\widetilde{g}\left(a^{2}+b^{2} i\right)=N_{K(i) / k(i)}\left(a^{2}+b^{2} i-\vartheta\right) \in k(i)^{2}$.

Theorem 4.12 Let $k$ be a hereditarily $\infty$-pythagorean field. Let $f \in k[X]$ be a monic polynomial such that every root of $f$ different from zero lies in $k(i) \backslash k$ and is of the form $\alpha_{0}^{2} \pm \alpha_{1}^{2} i$ with $\alpha_{0} \in k$ and $\alpha_{1} \in k^{\times}$. Then the function field $F$ of the curve $Y^{2}=f(X)$ has pythagoras number $p(F)=2$.

Proof Let $E=k(X)$, so that $F=E(\sqrt{f})$. Note that $f \in \sum E^{2} \cup X \sum E^{2}$. Let $g \in$ $k[X] \cap \sum F^{2}$ be a polynomial with only real roots. Then we have $g \in E^{\times} \cap \sum F^{2}=$ $\sum E^{2}+f \sum E^{2} \subseteq \sum E^{2}+X \sum E^{2}$. For any nonzero root $\alpha$ of $f$, we have $k(\alpha)=k(i)$ and thus $g(\alpha) \in k(\alpha)^{\times 2}$, by (4.11). In view of (4.9), this shows that $p(F)=2$.

Example 4.13 Let $k$ be a hereditarily $\infty$-pythagorean field. Then the function field of the curve $Y^{2}=X\left(X^{2}+1\right)$ over $k$ has pythagoras number 2 , by $(4.12)$.

Question 4.14 Let $k$ be a field with $-1 \notin k^{\times 2}$ and such that the function field of $Y^{2}=$ $X\left(X^{2}+1\right)$ has pythagoras number 2 . Then $k$ is hereditarily pythagorean, by (4.3). Is $k$ necessarily hereditarily $\infty$-pythagorean? 


\section{Henselian valued fields}

Let $\widetilde{k}$ denote the separable closure of $k$. If $k$ is of characteristic zero, in particular if $k$ is real, then $\widetilde{k}$ is just the algebraic closure of $k$. Given a valuation $v$ on $k$, we denote by $k_{v}$ the residue field, by $\mathcal{O}_{v}$ or $\mathcal{O}_{v, k}$ the corresponding valuation ring in $k$, and by $\Gamma_{v}$ or $\Gamma_{v, k}$ the value group of $v$ on $k$. Any valuation $v$ on $k$ can be extended to $k(X)$ in such way that $v\left(a_{0}+a_{1} X+\cdots+a_{n} X^{n}\right)=\min \left\{v\left(a_{0}\right), \ldots, v\left(a_{n}\right)\right\}$ for arbitrary $a_{0}, \ldots, a_{n} \in k, n \in \mathbb{N}$. This extension to $k(X)$, uniquely determined by this formula, is called the Gauss extension, and is again denoted by $v$. It has value group $\Gamma_{u, k(X)}=\Gamma_{v, k}$ and residue field $k_{v}(X)$. Obviously, the Gauss extension depends on the choice of the variable $X$, i.e. it is not stable under $k$ automorphisms of $k(X)$. For more details on extending valuations from $k$ to $k(X)$, we refer to $[13$, Sect. 2.2.1].

The next three statements are inspired by a simple argument we learned from $S$. Tikhonov, showing that $t X$ is a sum of 3 but not a sum of 2 squares in the function field of the curve $Y^{2}=(t X-1)\left(X^{2}+1\right)$ over $\mathbb{R}((t))$ (see also [16, (3.11)]). D. Leep gave us the idea how to generalise this example.

Proposition 5.1 Let $v$ be a valuation on $k$. Let $E=k(X)$ and $F=E(\sqrt{f})$ with $f \in$ $k[X]$ such that $v(f)=0$. If $n \in \mathbb{N}$ is such that $s\left(k_{v}\right) \geq 2^{n}$ and $\ell_{k_{v}(X)}(-\bar{f}) \geq 2^{n}$, then $v\left(E^{\times} \cap D_{F}\left(2^{n}\right)\right) \subseteq 2 \Gamma_{v}$. Moreover, if $k_{v}$ is real and $-\bar{f} \notin \sum k_{v}(X)^{2}$, then $v\left(E^{\times} \cap \sum F^{2}\right) \subseteq$ $2 \Gamma_{v}$.

Proof Let $n \in \mathbb{N}$ be such that $s\left(k_{v}\right) \geq 2^{n}$ and $\ell_{k_{v}(X)}(-\bar{f}) \geq 2^{n}$. So, the forms $2^{n} \times\langle 1\rangle$ and $\left(2^{n}-1\right) \times\langle 1\rangle \perp\langle\bar{f}\rangle$ are anisotropic over $k_{v}$. Therefore, we have $v\left(D_{E}\left(2^{n}\right)\right) \subseteq 2 \Gamma_{v}$ as well as $v\left(D_{E}(\varphi)\right) \subseteq 2 \Gamma_{v}$ for the quadratic form $\varphi=\langle f\rangle \perp\left(2^{n}-1\right) \times\langle 1\rangle$ over $k$. Since $E^{\times} \cap D_{F}\left(2^{n}\right)=D_{E}\left(2^{n}\right) \cdot D_{E}(\varphi)$ by (2.3), it follows that $v\left(E^{\times} \cap D_{F}\left(2^{n}\right)\right) \subseteq 2 \Gamma_{\nu}$. This proves the first part of the statement. The second claim follows immediately from the first.

Corollary 5.2 Let $v$ be a valuation on $k$. Let $F=k(X)(\sqrt{f})$ where $f \in k[X]$ is such that $v(f) \in 2 \Gamma_{v}$. Let $c \in k^{\times}$and $h \in k[X]$ be such that $f=c^{2} h$ and $v(h)=0$. Let $n \in \mathbb{N}$ be such that $s\left(k_{v}\right) \geq 2^{n}$ and $\ell_{k_{v}(X)}(-\bar{h}) \geq 2^{n}$. Then either $v\left(k(X)^{\times} \cap \sum F^{2}\right) \subseteq 2 \Gamma_{v}$ or $p(F)>2^{n}$.

Proof By the hypotheses and (5.1), we have $v\left(k(X)^{\times} \cap D_{F}\left(2^{n}\right)\right) \subseteq 2 \Gamma_{v}$. Thus, if $p(F) \leq 2^{n}$, i.e. $\sum F^{2}=D_{F}\left(2^{n}\right)$, then $v\left(k(X)^{\times} \cap \sum F^{2}\right) \subseteq 2 \Gamma_{v}$.

Theorem 5.3 Let $v$ be a valuation on $k$ with real residue field $k_{v}$. Let $t, c \in k^{\times}$with $0<$ $v(t) \leq v(c)$ and $v(t) \notin 2 \Gamma_{v}$. Let $h(X) \in \mathcal{O}_{v}[X]$ be a monic polynomial with only nonreal roots and let $n \in \mathbb{N}$ be such that $\ell_{k_{v}}(\bar{h}(X)) \geq 2^{n}$. Let $F$ be the function field of the curve $Y^{2}=(c X-1) h(X)$ over $k$. Then $F$ is real and $p(F)>2^{n}$.

Proof Note that $h$ is a sum of squares in $k[X]$. It is clear that $F$ is real. Let $b=2 t+t^{2}+c X$. Then $v(b)=v(t) \notin 2 \Gamma_{v}$, whence $b \notin D_{F}\left(2^{n}\right)$ by (5.1). However, $b=(1+t)^{2}+Y^{2} h^{-1}$ is a sum of squares in $F$. Therefore, $p(F) \geq \ell_{F}(b)>2^{n}$.

Assume now that the valuation $v$ on $k$ is henselian. Then $v$ has a unique extension to $\widetilde{k}$, which we also denote by $v$; the value group $\Gamma_{v, k}$ then is a subgroup of $\Gamma_{v, \widetilde{k}}$. Note that, if $k_{v}$ is hereditarily pythagorean, then so is $k$.

Often we will assume $k_{v}$, real closed, or at least hereditarily euclidean. The standard example for this situation is $k=\mathbb{R}((t))$, the field of Laurent series with real coefficients, 
where the valuation $v: k \rightarrow \mathbb{Z} \cup\{\infty\}$ is trivial on $\mathbb{R}$ and takes $t$ to 1 ; in this case, we have $k_{v}=\mathbb{R}$ and $\Gamma_{v, k}=\mathbb{Z}$.

Lemma 5.4 Let $v$ be a henselian valuation on $k$. Let $g \in k[X]$ and $\alpha \in \tilde{k}$ with $v(\alpha)=0$. If for every root $\vartheta \in \tilde{k}$ of $g$ with $v(\vartheta)=0$ one has $\bar{\vartheta} \neq \bar{\alpha}$ in $\bar{k}_{v}$, then $v(g(\alpha))=v(g(X))$.

Proof Let $\vartheta \in \tilde{k}$ be a root of $g$. We claim that $v(X-\vartheta)=v(\alpha-\vartheta)$. This claim obviously implies the statement. Note that $v(X)=v(\alpha)=0$. Therefore, the claim is obvious if $v(\vartheta) \neq 0$. Suppose now that $v(\vartheta)=0$. Then it is clear that $v(X-\vartheta)=0$ and $v(\alpha-\vartheta) \geq 0$. Now $\bar{\vartheta} \neq \bar{\alpha}$ yields the equality $v(\alpha-\vartheta)=0=v(X-\vartheta)$.

Let $v$ be a henselian valuation on $k$. We say that $a \in \widetilde{k}$ has good reduction (with respect to $v$ and $k)$ if $v(a)=0$ and $\left[k_{v}(\bar{a}): k_{v}\right]=[k(a): k]$.

Theorem 5.5 Let $v$ be a henselian valuation on $k$ with hereditarily euclidean residue field $k_{v}$. Let $E=k(X)$ and $F=E(\sqrt{f})$ where $f \in k[X]$ is a nonzero polynomial such that all nonreal roots of $f$ have good reduction with respect to $v$. Then $E^{\times} \cap \sum F^{2} \cap v^{-1}\left(2 \Gamma_{v}\right) \subseteq D_{F}(2)$ and $\left|\sum F^{2} / D_{F}(2)\right| \leq\left|\Gamma_{v} /\left(2 \Gamma_{v} \cup\left(v(f)+2 \Gamma_{v}\right)\right)\right|$.

Proof Let $g \in E^{\times} \cap \sum F^{2}$ with $v(g) \in 2 \Gamma_{v}$ be given. Since $E$ is the quotient field of $k[X]$, in order to show that $g \in D_{F}(2)$, we may assume that $g$ is a polynomial in $X$ with no multiple roots. Since for the nonreal part $g_{*}$ of $g$ we have $g_{*} \in D_{E}(2) \cap v^{-1}\left(2 \Gamma_{v}\right)$, we may assume $g_{*}=1$, that is, $g$ has only real factors. Let $\alpha$ be a nonreal root of $f$. Since, by the hypothesis, $\alpha$ has good reduction with respect to $v$, we have $v(\alpha)=0, k(\alpha) \supseteq k(i)$, and $k_{v}(\bar{\alpha}) \supseteq k_{v}(i)$. On the other hand, for any root $\vartheta$ of $g$ with $v(\vartheta)=0$, the residue class $\bar{\vartheta}$ generates a real extension of $k_{v}$, so that in particular $\bar{\vartheta} \neq \bar{\alpha}$. Therefore (5.4) yields $v(g(\alpha))=v(g)$. Note that $k_{v}(\alpha)$ is quadratically closed, because $k_{v}$ is hereditarily euclidean. Therefore, $k(\alpha)^{\times 2}=k(\alpha) \cap v^{-1}\left(2 \Gamma_{v, k(\alpha)}\right)$. Since $v(g) \in 2 \Gamma_{v}$, it follows that $g(\alpha)$ is a square in $k(\alpha)$. Since this holds for any nonreal root $\alpha$ of $f$, using (4.8) we conclude that $g \in$ $D_{E}(\langle 1, f\rangle) \subseteq D_{F}(2)$. This shows the inclusion claimed in the statement. Since $f \in F^{\times 2}$ we even obtain that $E^{\times} \cap \sum F^{2} \cap v^{-1}\left(2 \Gamma_{v} \cup\left(v(f)+2 \Gamma_{v}\right)\right) \subseteq D_{F}(2)$. Observe that the quotient $\sum F^{2} \cap E^{\times} / \sum F^{2} \cap E^{\times} \cap v^{-1}\left(2 \Gamma_{v} \cup\left(v(f)+2 \Gamma_{v}\right)\right)$ injects into $\Gamma_{v} /\left(2 \Gamma_{v} \cup\left(v(f)+2 \Gamma_{v}\right)\right)$. By the last inclusion, the same quotient surjects onto $\sum F^{2} \cap E^{\times} / D_{F}(2) \cap E^{\times}$. Moreover, $D_{F}(2) \cap E^{\times}=D_{F}(2) \cap \sum F^{2} \cap E^{\times}$, thus $\left(\sum F^{2} \cap E^{\times}\right) /\left(D_{F}(2) \cap E^{\times}\right)$is isomorphic to $\left(\sum F^{2} \cap E^{\times}\right) D_{F}(2) / D_{F}(2)$. Now recall that $\sum F^{2}=D_{F}(2)\left(E^{\times} \cap \sum F^{2}\right)$, by (3.8) applied with $n=0$. This yields the claimed estimate.

Corollary 5.6 Assume that $k$ carries a henselian valuation $v$ with hereditarily euclidean residue field $k_{v}$. Let $F=k(X)(\sqrt{f})$ where $f \in k[X]$ is a polynomial with $v(f) \in 2 \Gamma_{v}$ and such that all nonreal roots of $f$ are of good reduction with respect to $v$. We may write $f=c^{2} h$ with $c \in k^{\times}$and $h \in k[X]$ such that $v(h)=0$. If $-\bar{h}$ is not a sum of squares in $k_{v}[X]$, then $p(F)=2$.

Proof Replacing $f$ by $h$, which does not change the function field $F$, we may assume that $v(f)=0$ and that $-\bar{f}$ is not a square in $k_{v}[X]$, thus also not in $E=k(X)$. Then $v\left(E^{\times} \cap\right.$ $\left.\sum F^{2}\right) \subseteq 2 \Gamma_{v}$ by (5.1) and therefore (5.5) yields the inclusion $E^{\times} \cap \sum F^{2} \subseteq D_{F}(2)$. Since $p(E)=2$, it follows that $\sum F^{2}=D_{F}(2)\left(E^{\times} \cap \sum F^{2}\right)=D_{F}(2)$. Therefore, $p(F)=2$.

We now focus on function fields over fields $k$ carrying a henselian valuation with value group $\mathbb{Z}$ and with hereditarily euclidean residue field. The typical example for such $k$ is still 
the field $\mathbb{R}((t))$. The aim is to determine the values of $u(F), p(F)$, and $\left|\sum F^{2} / F^{\times 2}\right|$ for function fields of hyperelliptic curves $F / k$, if possible. To deal with the $u$-invariant, we need some preparation.

Proposition 5.7 Let $k$ be a field carrying a henselian valuation $v$ with value group $\mathbb{Z}$ and such that the residue field $k_{v}$ is hereditarily quadratically closed of characteristic zero. Let $F$ be a function field in one variable over $k$. Then $u(F)=4$.

Proof Observe that $k$ satisfies the assumption of $(4.5)$, whence $u(F) \geq 4$. To prove the converse inequality, we first assume that $k_{v}$ is algebraically closed of characteristic zero. Then $k$ is a $\mathcal{C}_{1}$-field by [10], and therefore $F$ is a $\mathcal{C}_{2}$-field by Tsen-Lang Theory, which implies that $u(F) \leq 4$.

Suppose now that $k_{v}$ is hereditarily quadratically closed of characteristic zero. Then $k_{v}$ has no finite extensions of even degree. Let $k^{\prime}$ be the maximal unramified extension of $k$ with respect to $v$, and let $k_{v}^{\prime}$ be the corresponding extension of the residue field. Then every element of $k^{\prime}$ has odd degree over $k$ and $k_{v}^{\prime}$ is the algebraic closure of $k$. Let $F^{\prime}=F k^{\prime}$. Then it follows from [15, Chap. III, (6.2)] that also every element of $F^{\prime}$ has odd degree over $F$. Using Springer's Theorem [9, Chap. VII, (2.7)], and the previous case, we obtain that $u(F) \leq u\left(F^{\prime}\right) \leq 4$.

Corollary 5.8 Let $k$ be a field carrying a henselian valuation $v$ with value group $\mathbb{Z}$ and such that the residue field $k_{v}$ is hereditarily euclidean. Let $F$ be a function field in one variable over $k$. If $\left|\sum F^{2} / F^{\times 2}\right| \leq 2$, then $u(F)=4$.

Proof Since $k_{v}(i)$ is hereditarily quadratically closed of characteristic zero, we have $u(F(i))=4$ by (5.7). With $\left|\sum F^{2} / F^{\times 2}\right| \leq 2$ we thus obtain from (2.2) that $u(F)$ $u(F(i))=4$. On the other hand, $u(F) \geq 4$ by (4.5).

Theorem 5.9 Let $k$ be a field carrying a henselian valuation $v$ with value group $\mathbb{Z}$ and such that the residue field $k_{v}$ is hereditarily euclidean. Let $F$ be the function field of a hyperelliptic curve $Y^{2}=f(X)$ over $k$ where $f \in k[X]$ has at most one nonreal irreducible factor. Then $u(F)=4$.

Proof Since $k_{v}(i)$ is hereditarily quadratically closed of characteristic zero, we have $u(F(i))=4$ by (5.7). By the assumptions on $k$ and $v$, we have $K^{\times} / K^{\times 2} \cong \mathbb{Z} / 2 \mathbb{Z}$ for any finite nonreal extension $K / k$. Therefore, with the assumption on $f$, applying (5.5) we obtain that $\left|\sum F^{2} / D_{F}(2)\right| \leq 2$. Hence, $u(F) \leq u(F(i))=4$ by (2.2). On the other hand, $u(F) \geq 4$ by $(4.5)$.

Corollary 5.10 Let $k$ be a field carrying a henselian valuation $v$ with value group $\mathbb{Z}$ and such that the residue field $k_{v}$ is hereditarily euclidean. Let $F$ be the function field of a conic or an elliptic curve over $k$. Then $u(F)=4$.

Proof This is a special case of (5.9).

The following result generalises and extends [16, Theorem 1.4].

Theorem 5.11 Let $v$ be a henselian valuation on $k$ with value group $\mathbb{Z}$ and with hereditarily euclidean residue field $k_{v}$. Let $f \in k[X]$ be a nonzero polynomial such that all nonreal roots of $f$ have good reduction with respect to $v$. Let $F$ be the function field of the curve $Y^{2}=f(X)$ over $k$. Then

$$
\left|\sum F^{2} / D_{F}(2)\right| \leq 2 \text { and } u(F)=4
$$


Moreover, $p(F)=2$ except possibly in the case where $v(f) \in 2 \mathbb{Z}$ and, writing $f=c^{2} h$ with $c \in k^{\times}$and $h \in k[X]$ of valuation $v(h)=0$, the residue polynomial $-\bar{h}$ is a sum of squares in $k_{v}[X]$.

Proof In the case where $v(f) \in 2 \mathbb{Z}$, except for the $u$-invariant this is a reformulation of (5.6). Otherwise, we have $v(f) \notin 2 \mathbb{Z}$ and then the inequality in (5.5) actually yields that $\sum F^{2}=D_{F}(2)$, thus $p(F)=2$. In any case, using (5.8) we obtain that $u(F)=4$.

We now give an example of a function field of a hyperelliptic curve over $k((t))$, where neither (5.9) nor (5.11) applies and where we have not been able to decide whether the $u$-invariant is 4 .

Example 5.12 Let $F$ be the function field of $Y^{2}=-\left(X^{2}+1\right)\left(X^{2}+t^{2}\right)$ over $k=\mathbb{R}((t))$. Then $F$ is nonreal with $-1 \notin F^{\times 2}$. We claim that $\left|\sum F^{2} / D_{F}(2)\right|=4$, in particular $p(F)=$ $s(F)+1=3$. In view of (3.10), it is sufficient to show that none of $t, X$, and $t X$ lies in $D_{F}(2)$.

We write $f=-\left(X^{2}+1\right)\left(X^{2}+t^{2}\right)$ and consider the canonical extension to $E=k(X)$ of the valuation $v: k \rightarrow \mathbb{Z} \cup\{\infty\}$ associated to $t$. It follows from (5.1) that $v\left(E^{\times} \cap D_{F}(2)\right) \subseteq 2 \mathbb{Z}$. Therefore, $t, t X \notin D_{F}(2)$.

Assume now that $X \in D_{F}(2)$. Then the quadratic form $\langle 1, f,-X,-X\rangle$ is isotropic over $k(X)$. By [12, Chap. 1, (2.3)], this implies that

$$
X^{4}+\left(1+t^{2}\right) X^{2}+t^{2}=-f=g^{2}-X\left(h_{1}^{2}+h_{2}^{2}\right)
$$

with $g, h_{1}, h_{2} \in k[X]$. Comparing the degrees of both sides shows that $g$ is a quadratic polynomial with leading coefficient \pm 1 and constant coefficient $\pm t$, while $h_{1}$ and $h_{2}$ are linear or constant. We write $g(X)= \pm X^{2}+b X \pm t$ with $b \in k$. Comparing the linear coefficients, we obtain $\pm 2 b t=h_{1}(0)^{2}+h_{2}(0)^{2}$ and thus $v(b) \notin 2 \mathbb{Z}$, while comparing coefficients in degree 3 yields on the contrary that $v(b) \in 2 \mathbb{Z}$. Therefore, we have $X \notin D_{F}(2)$, as claimed.

The following example shows that $p(F)=2$ may occur in the case that is left open in (5.11). Moreover, it shows that $p(F)=s(F)$ is possible for a nonreal function field of a hyperelliptic curve over $\mathbb{R}((t))$.

Example 5.13 Let $F$ be the function field of $Y^{2}=-\left(X^{2}+1\right)\left(X^{2}+1+t\right)$ over $\mathbb{R}((t))$. Then $F$ is nonreal with $-1 \notin F^{\times 2}$. By (5.5) we have the inclusion $E^{\times} \cap v^{-1}(2 \mathbb{Z}) \subseteq D_{F}$ (2). It follows that $F^{\times}=D_{F}(2) \cup-t D_{F}(2)$. But $-t\left(X^{2}+1\right)=Y^{2}+\left(X^{2}+1\right)^{2}$ in $F$, showing that $-t$ is also a sum of two squares in $F$. Therefore, we have $p(F)=2=s(F)$.

Consider now the function field $F$ of an elliptic curve over $k=\mathbb{R}((t))$. Thus $F$ is given by a curve $Y^{2}=f(X)$ where $f \in k[X]$ is of degree 3 . If $f$ has only real factors then $p(F)=2$ by $(4,10)$. In the remaining case, $f$ is a product of a linear polynomial with an irreducible quadratic polynomial with root field $k(i)$. After a linear substitution we are then in the situation where the following theorem allows to decide whether $p(F)=2$ or $p(F)>2$.

Theorem 5.14 Let $k$ be a real field carrying a henselian valuation $v$ with value group $\mathbb{Z}$ and with hereditarily euclidean residue field $k_{v}$. Let $f \in k[X]$ be a polynomial of degree 3 with $f(0)=0$ and let $F$ be the function field of the elliptic curve $Y^{2}=f(X)$. If $v(f) \in 2 \mathbb{Z}$ and if $f$ has a root $\alpha_{0}+i \alpha_{1}$ in $k(i)$ with $\alpha_{0}, \alpha_{1} \in k^{\times}$such that $v\left(\alpha_{0}\right)<v\left(\alpha_{1}\right)$ and $-\alpha_{0} \in k^{\times 2}$, then $p(F)$ is either 3 or 4 . In any other case $p(F)=2$. 
Proof Recall that $2 \leq p(F) \leq 4$ by (4.2). If $v(f) \notin 2 \mathbb{Z}$ or if $f$ has no root in $k(i) \backslash k$, then $p(F)=2$, by (5.11) and (4.10). In the remaining case we may assume that $f$ is monic and write $f=X\left(\left(X-\alpha_{0}\right)^{2}+\alpha_{1}^{2}\right)$ with $\alpha_{0}, \alpha_{1} \in k$ and $\alpha_{1} \neq 0$. Substituting $X=\alpha_{1} X^{\prime}+\alpha_{0}$ and $Y=\alpha_{1} Y^{\prime}$ shows that $F$ is also the function field of the curve $Y^{2}=g(X)$ for $g=\left(\alpha_{1} X+\alpha_{0}\right)\left(X^{2}+1\right)$. Now, again by (5.11), we readily obtain $p(F)=2$ for the cases where $v\left(\alpha_{0}\right) \geq v\left(\alpha_{1}\right)$ or $-\alpha \notin \sum k^{2}=k^{\times 2}$. On the other hand, if $v\left(\alpha_{0}\right)<v\left(\alpha_{1}\right)$ and $-\alpha_{0} \in k^{\times 2}$, then $p(F)>2$ follows from (5.3).

Question 5.15 Let $h \in \mathbb{R}[X]$ be a nonconstant square-free polynomial with no roots in $\mathbb{R}$. Let $F$ be the function field of the curve $Y^{2}=(t X-1) h(X)$ over $\mathbb{R}((t))$. Then $3 \leq p(F) \leq 4$ by (5.3) and (4.2). What is the exact value of $p(F)$ ?

Acknowledgments The results in this article grew out of discussions the authors had during the past three years. We would like to thank David Leep, Sergey Tikhonov, and Vyacheslav Yanchevskil for stimulating discussions around the topic of this article. Furthermore, the project turned out to be a very good reason for one author to visit Ghent regularly and for the other to visit Lausanne and Konstanz. We are grateful to the institutes, including the École Polytechnique Fédérale de Lausanne, for their hospitality. We further acknowledge support from the European RTN Network 'Algebraic K-Theory, Linear Algebraic Groups, and Related Structures' (HPRN-CT-2000-00287) and the Swiss National Science Foundation (Grant No. 200020100229/1).

\section{References}

1. Becher, K.J.: On fields of $u$-invariant 4. Arch. Math. 86, 31-35 (2006)

2. Becher, K.J.: Minimal weakly isotropic forms. Math. Z. 252, 91-102 (2006)

3. Becker, E.: Hereditarily-pythagorean fields and orderings of higher level. Monografias de Matemática Vol. 29. Instituto de matematica pura e aplicada, Rio de Janeiro (1978)

4. Elman, R., Lam, T.Y.: Quadratic forms and the $u$-invariant, $I$. Math. Z. 131, 283-304 (1973)

5. Elman, R., Lam, T.Y.: Quadratic forms under algebraic extensions. Math. Ann. 219, $21-42$ (1976)

6. Elman, R., Wadsworth, A.R.: Hereditarily quadratically closed fields. J. Algebra 111, 475-482 (1987)

7. Hoffmann, D.W.: Pythagoras numbers of fields. J. Am. Math. Soc. 12, 839-848 (1999)

8. Hoffmann, D.W.: Isotropy of quadratic forms and field invariants. In: Bayer-Fluckiger, E. et al (eds.) Quadratic Forms and their Applications. Proceedings of the conference, University College Dublin, Ireland, July 5-9, 1999. American Mathematical Society (AMS), Providence, RI. Contemp. Math. 272 73-102 (2000)

9. Lam, T.Y.: Introduction to quadratic forms over fields. Graduate Studies in Mathematics, vol. 67. American Mathematical Society, Providence, RI (2005)

10. Lang, S.: On quasi-algebraic closure. Ann. Math. 55, 373-390 (1952)

11. Pfister, A.: Zur Darstellung definiter Funktionen als Summe von Quadraten. Invent. Math. 4, 229-237 (1967)

12. Pfister, A.: Quadratic forms with applications to algebraic geometry and topology. LMS Lect. Notes Series Vol. 217. Cambridge University Press, Cambridge, UK (1995)

13. Engler, A.J., Prestel, A.: Valued fields. Springer Monographs in Mathematics. Springer, Berlin (2005)

14. Scharlau, W.: Quadratic and hermitian forms. Grundlehren der Mathematischen Wissenschaften, vol. 270. Springer, Berlin (1985)

15. Suchtenoth, H.: Algebraic function fields and codes. Universitext. Springer, Berlin (1993)

16. Tikhonov, S.V., Van Geet, I., Yanchevskii, V.I.: Pythagoras numbers of function fields of hyperelliptic curves with good reduction. Manuscripta Math. 119, 305-322 (2006)

17. Tikhonov, S.V., Yanchevski, V.I.: Pythagoras numbers of function fields of conics over hereditarily pythagorean fields. Dokl. Nats. Akad. Nauk Belarusi 47(2), 5-8 (2003) 